\title{
Diagnostics of solar flare reconnection
}

\author{
M. Karlický and M. Bárta \\ Astronomical Institute, Academy of Sciences of the Czech Republic, 25165 Ondřejov, Czech Republic
}

Received: 11 August 2004 - Accepted: 15 October 2004 - Published: 1 November 2004

Part of Special Issue "Nonlinear processes in solar-terrestrial physics and dynamics of Earth-Ocean-System"

\begin{abstract}
We present new diagnostics of the solar flare reconnection, mainly based on the plasma radio emission. We propose that the high-frequency (600-2000 MHz) slowly drifting pulsating structures map the flare magnetic field reconnection. These structures correspond to the radio emission from plasmoids which are formed in the extended current sheet due to tearing and coalescence processes. An increase of the frequency drift of the drifting structures is interpreted as an increase of the reconnection rate. Using this model, time scales of slowly drifting pulsating structure observed during the 12 April 2001 flare by the Trieste radiopolarimeter with high time resolution $(1 \mathrm{~ms})$ are interpreted as a radio manifestation of electron beams accelerated in the multi-scale reconnection process. For short periods Fourier spectra of the observed structure have a power-law form with power-law indices in the 1.3-1.6 range. For comparison the 2-D MHD numerical modeling of the multi-scale reconnection is made and it is shown that Fourier spectrum of the reconnection dissipation power has also a power-law form, but with power-law index 2 . Furthermore, we compute a time evolution of plasma parameters (density, magnetic field etc) in the 2-D MHD model of the reconnection. Then assuming a plasma radio emission from locations, where the "doubleresonance" instability generates the upper-hybrid waves due to unstable distribution function of suprathermal electrons, we model radio spectra. Effects of the MHD turbulence are included. The resulting spectra are compared with those observed. It is found, that depending on model parameters the lace bursts and the decimetric spikes can be reproduced. Thus, it is shown that the model can be used for diagnostics of the flare reconnection process. We also point out possible radio signatures of reconnection outflow termination shocks. They are detected as type II-like herringbone structures in the $200-700 \mathrm{MHz}$ frequency range. Finally, we mention $\mathrm{H} \alpha$ spectra of the 18 September 1995 eruptive prominence which indicate the bi-directional plasma flow as expected in the reconnection process.
\end{abstract}

Correspondence to: M. Karlický

(karlicky@asu.cas.cz)

\section{Introduction}

The reconnection of magnetic field lines plays the key role in complex solar active processes which manifest themselves as various observational dynamic phenomena - such as solar flares, plasmoid ejecta and CMEs - see e.g. Priest and Forbes (2000). However, it is very difficult to detect the reconnection process itself, our information comes only from consequent processes such as accelerated particles, mass motions, plasma heating etc. Therefore, any further observations related more directly to the magnetic reconnection would be of great importance.

Up to now the most of observational evidence of the magnetic reconnection comes from the soft X-ray and EUV observations. For example, Yokoyama et al. (2001) presented the 18 March 1999 event as an evidence of the reconnection inflow. Based on this observation they derived the reconnection rate as $M_{A}=0.001-0.03$, where $M_{A}$ is an Alfvén Mach number of the inflow. Furthermore, Asai et al. (2004) showed downflow motions associated with impulsive nonthermal emission observed during the 23 July 2002 solar flare. This result implies that the downflow motions occurred when strong magnetic energy was released and that they are correlated with the reconnection outflows. Bi-directional plasma flows, which characterize the magnetic reconnection, are reported also in the paper by Curdt et al. (1998) where the SUMER/SOHO spectra taken in the corona above the active regions are analyzed.

In the present paper, we summarize new possibilities, mainly based on the radio plasma emission, how to diagnose the magnetic field reconnection. First, the high-frequency slowly drifting pulsating structures, which according to our model map the reconnection process, are described. Then we present the unified model of lace bursts and narrowband $\mathrm{dm}$-spikes, which enables us to diagnose the reconnection plasma outflows. The radio plasma emission from the reconnection outflow termination shocks is mentioned. Finally, it is shown that the $\mathrm{H} \alpha$ spectra can also indicate bi-directional reconnection flows. 

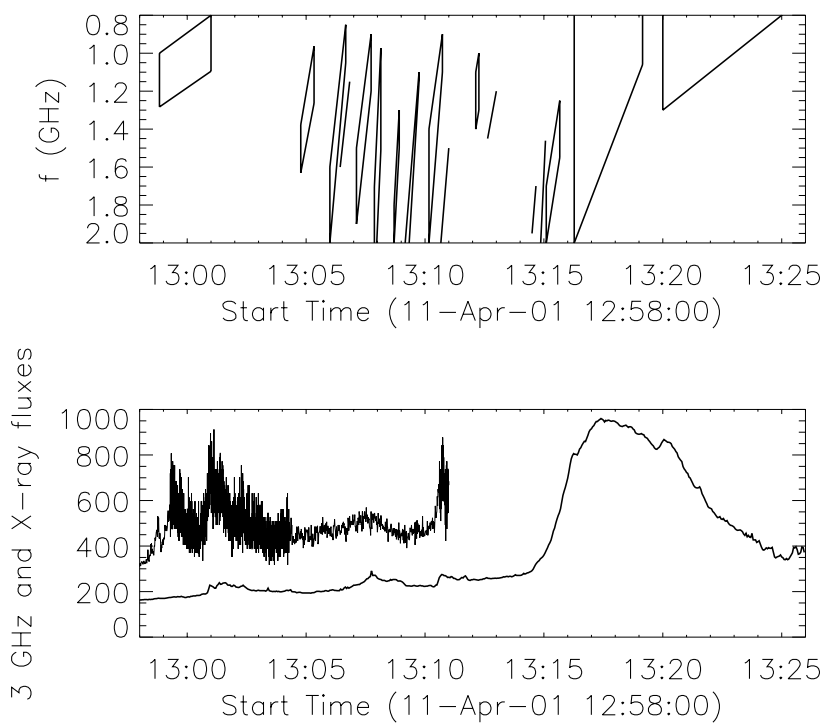

Fig. 1. Time evolution of high-frequency slowly drifting structures (outlines of emission structures in the time-frequency plane) (top), and $3 \mathrm{GHz}$ radio and HXRS (Fárník et al. 2001) 19-29 keV fluxes (in arbitrary units) (bottom) observed during the 11 April 2001 flare. Due to spacecraft night, the HXRS observation ended at 13:11 UT; after 13:04 UT the time resolution of the observations was changed from $0.2 \mathrm{~s}$ to $1 \mathrm{~s}$.

\section{High-frequency slowly drifting pulsating structures mapping the flare magnetic reconnection}

Slowly drifting pulsating structures, observed at the beginning of some eruptive solar flares in the $0.6-2.0 \mathrm{GHz}$ frequency range, have been found to be radio signatures of plasmoid ejection (Karlický and Odstrčil, 1994; Kliem et al., 2000; Khan et al., 2002; Karlický et al., 2002).

Hudson et al. (2001) identified a rapidly moving hard X-ray source, observed by the HXT (Hard X-ray Telescope) of the "Yohkoh" satellite, associated with the moving microwave source and the plasmoid ejection seen in images observed by the SXT (Soft X-ray Telescope) on board "Yohkoh". An association with a high-frequency slowly drifting burst was also reported.

Similarly, Kundu et al. (2001) identified two moving "Yohkoh" soft X-ray ejecta associated with moving decimetric/metric radio sources observed by the Nancay radioheliograph.

Based on the magnetohydrodynamic (MHD) numerical simulations, Kliem et al. (2000) suggested that each individual burst in the slowly drifting pulsating structure is generated by superthermal electrons, accelerated in the peak of the electric field in the quasi-periodic regime of the magnetic field reconnection. Furthermore, the global slow negative frequency drift of the structure was explained by a plasmoid propagation upwards in the solar corona towards lower plasma densities.

In the following, first, a unique example of the 11 April 2001 radio spectrum with a series of slowly drifting struc-

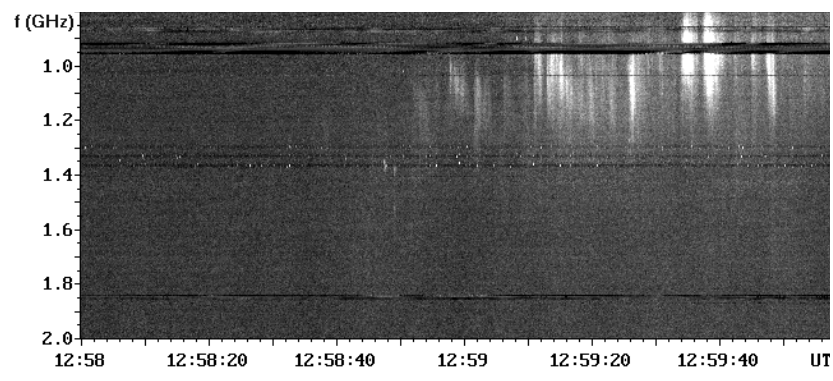

Fig. 2. High-frequency slowly drifting structure observed by the Ondřejov radiospectrograph at the very beginning of the 11 April 2001 flare, at 12:58 UT-13:00 UT.

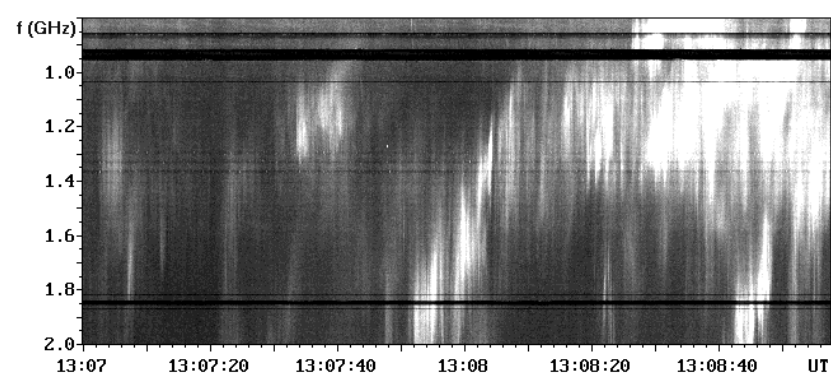

Fig. 3. Example of high-frequency slowly drifting structures observed by the Ondřejov radiospectrograph in 11 April 2001 at 13:07 UT-13:09 UT.

tures is shown. Namely, in most cases only one drifting pulsating structure (DPS) is observed. Then the 12 April 2001 event is analyzed from the point of view of time scales in the reconnection process.

\subsection{The 11 April 2001 event}

On 11 April 2001 the GOES satellite observed a soft X-ray event which started at 12:56 UT, with maximum at 13:26 UT and end at 13:49 UT. This event was classified as M2.3 and localized in the active region NOAA AR 9415. According to $\mathrm{H} \alpha$ observations the $1 \mathrm{~F}$ flare started at 13:01, maximum at 13:21 UT, and end at 14:23 UT; in the position S22W27.

As can be seen in Figs. 1, 2, and 3, a rare series of highfrequency slowly drifting structures was observed during the 11 April 2001 flare in the $0.8-2.0 \mathrm{GHz}$ frequency range by the Ondřejov radiospectrograph (Jiřička et al., 1993). Usually only one such a structure is recorded at the beginning of some eruptive flares. The slowly drifting structure shown in Fig. 2, which was recorded at the very beginning of this flare, can be considered as an example of such typical cases. In a relatively narrow frequency range it consists of many fast drift radio bursts with the characteristic repetition time of seconds. The structure as a whole drifts slowly towards lower frequencies.

During this event we recorded a series of slowly drifting structures. Their global evolution during the flare is presented schematically in the upper part of Fig. 1. For comparison, the time evolution of the $3 \mathrm{GHz}$ radio and HXRS (Fárník et al., 2001) 19-29 keV X-ray flux are added in 
FLARE SHOCK

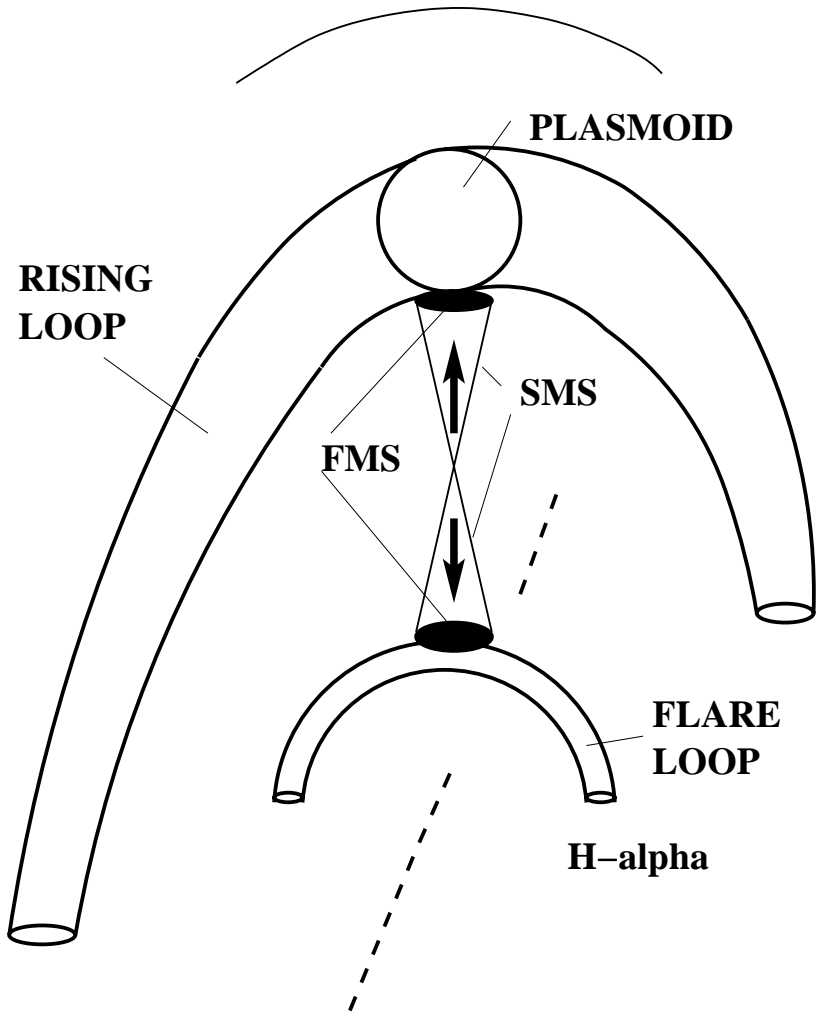

Fig. 4. Standard model of the dynamic (eruptive) solar flare. Between rising and flare loops current sheet is formed and here reconnection takes place. The reconnection outflows are indicated by arrows. SMS means slow magnetosonic shock and FMS fast magnetosonic (termination) shock

the bottom panel of Fig. 1. The radio phenomenon started at 12:58:50 UT with drifting structure having an instantaneous bandwidth of $400 \mathrm{MHz}$ and a relatively slow global frequency drift $\left(-1.5 \mathrm{MHz} \mathrm{s}^{-1}\right)$. Then in the time interval 13:04-13:12 UT a group of drifting structures having higher frequency drifts $\left(-12--45 \mathrm{MHz} \mathrm{s}^{-1}\right)$ followed. The repetition time of these drifting structures was about $45 \mathrm{~s}$. In this frequency range the activity ended with another group of drifting structures with drifts changing from $-45 \mathrm{MHz} \mathrm{s}^{-1}$ to $-1.2 \mathrm{MHz} \mathrm{s}^{-1}$ at the end.

All drifting structures in this flare were composed of many fast drifting quasi-periodic features (bursts) having characteristic time $\leq 1 \mathrm{~s}$, usually with very high (not measurable) frequency drifts. Their drifts were measurable only in a few cases, as for example in the initial drifting structure (Fig. 2), where pulses with frequency drifts of $-200-+450 \mathrm{MHz} \mathrm{s}^{-1}$ can be recognized.

A very interesting situation can be seen in the radio spectrum presented in Fig. 3, where several drifting structures are shown. For example one structure (at 13:07:52 UT13:09:00 UT, with bandwidth $400 \mathrm{MHz}$ ) drifts towards lower frequencies with about $-45 \mathrm{MHz} \mathrm{s}^{-1}$ and then its drift stops and further structures drift to and over this stopped structure (e.g. at 13:08:50 UT).

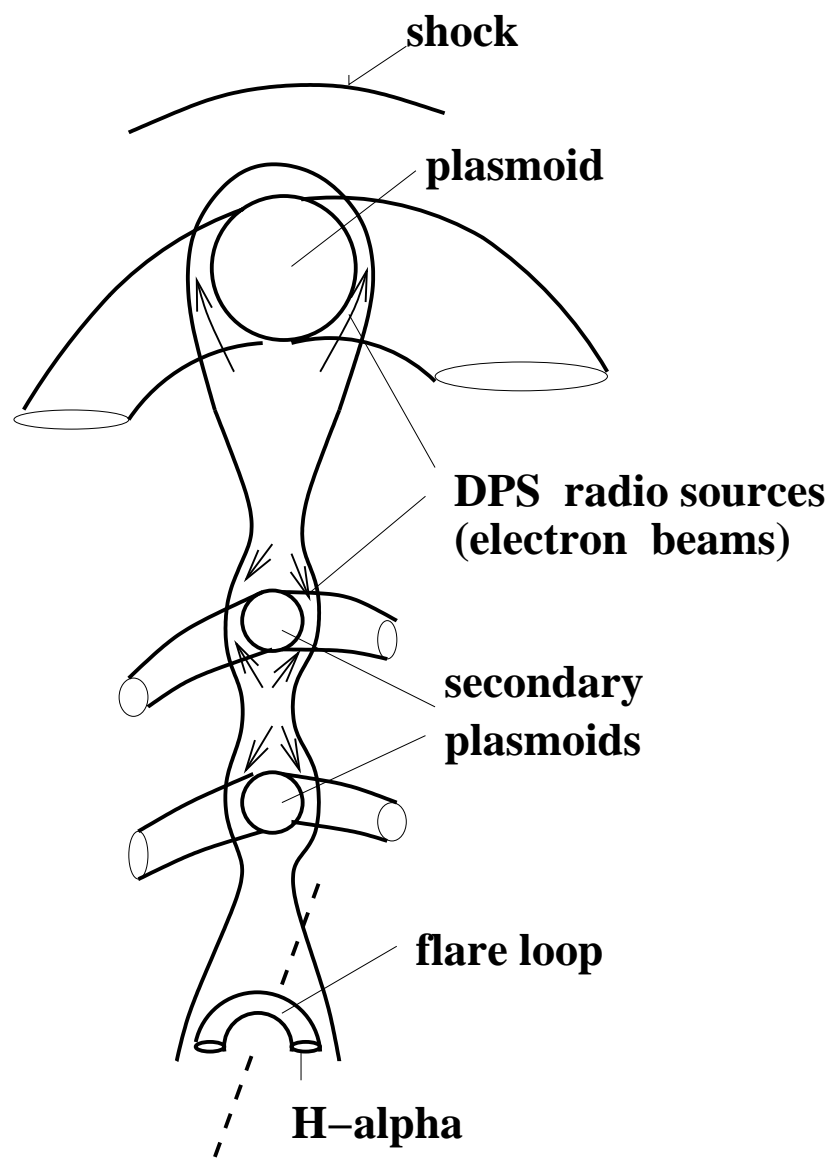

Fig. 5. Flare scenario: Due to tearing instability secondary plasmoids are generated. All these plasmoids can interact. DPS means drifting pulsating structure.

\subsubsection{Interpretation}

In the interpretation of the present event we start from the model proposed by Kliem et al. (2000). We interpret the initial drifting structure as plasma radio emission generated by electron beams which are injected into the plasmoid (Fig 4) during a quasi-periodic reconnection process in the current sheet below the plasmoid. The model enables us to estimate the electron plasma density in the initial plasmoid as, approximately, $n_{e}=1.77 \times 10^{10} \mathrm{~cm}^{-3}$ (assuming the plasma radio emission at the fundamental electron plasma frequency). According to the model (Ohyama and Shibata, 1998), the reconnection outflows can reach velocities comparable with the Alfvén speed, thus we expect that these outflows are in a turbulent state.

The 11 April 2001 flare is unique due to the series of drifting structures. In principle, in the dynamic reconnection in the current sheet, a set of plasmoids can be formed as a result of the tearing and the coalescence instabilities (Kliem et al., 2000). It was proposed that these processes have a cascading form: secondary tearing, tertiary tearing, and so on, always on shorter and shorter spatial scales (Tanuma et al., 2001; Shibata and Tanuma, 2001). Furthermore the plasmoids formed can merge into larger plasmoids. Tanuma et 


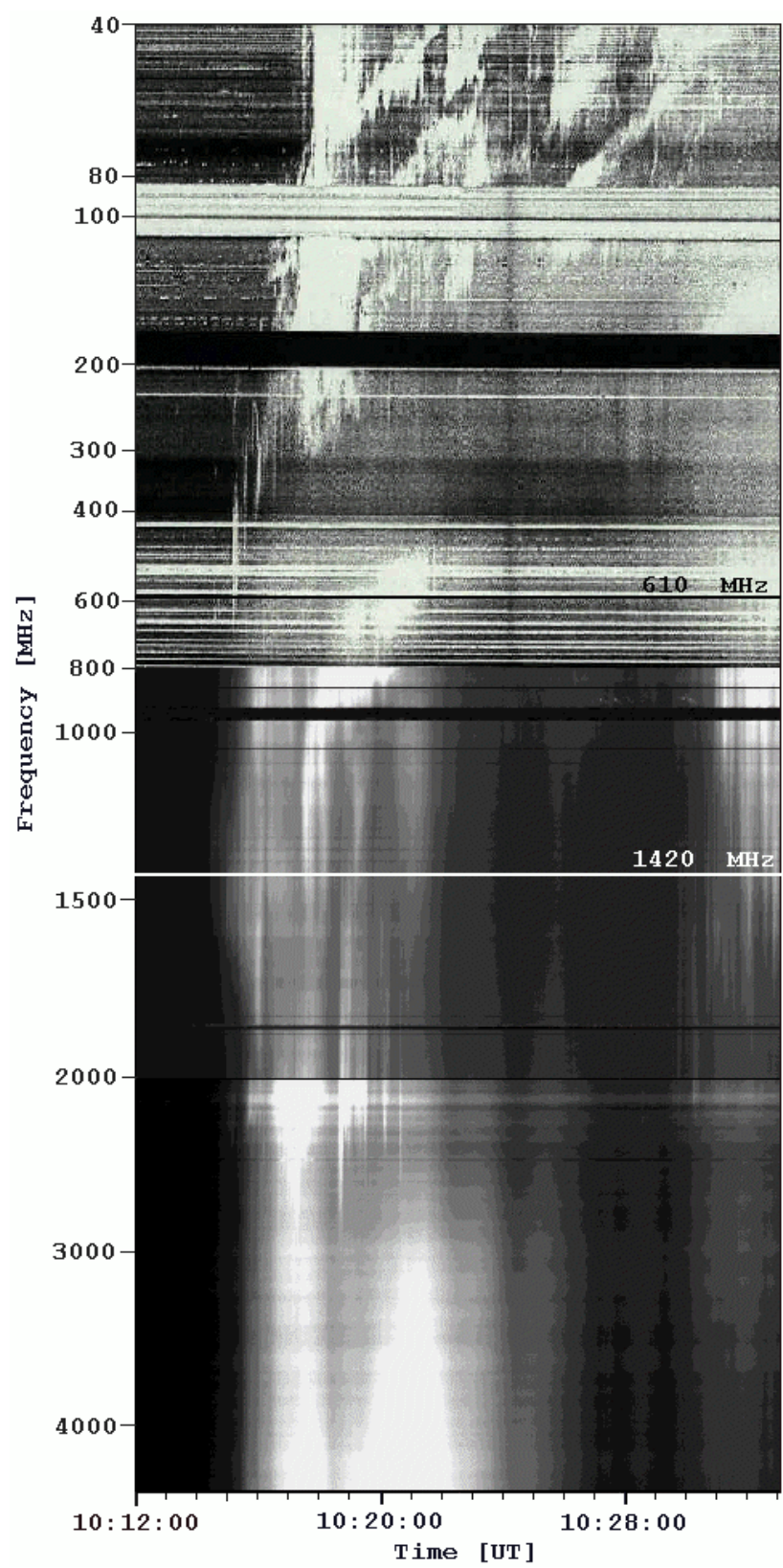

Fig. 6. The 40-4500 MHz radio spectrum observed during the 12 April 2001 event by the Potsdam (40-800 MHz range, courtesy by A. Klassen) and the Ondřejov radiospectrographs, showing the slowly drifting pulsating structure at 10:17:20 UT-10:22:00 UT in the 450-1500 MHz range and the type II radio burst at 10:17 UT10:33 UT in the 40-300 MHz range. The Trieste observing frequencies (610 and $1420 \mathrm{MHz}$ ) are shown by horizontal lines (black and white, respectively).

al. (2001) also showed that an increase of the velocity of the plasmoid ejection leads to an increase of the reconnection rate in the reconnection process.

We use these facts for the interpretation of the observed series of slowly drifting structures. We propose that each drifting structure corresponds to some plasmoids (see Fig. 5). Electron beams are accelerated in localized current sheets be-

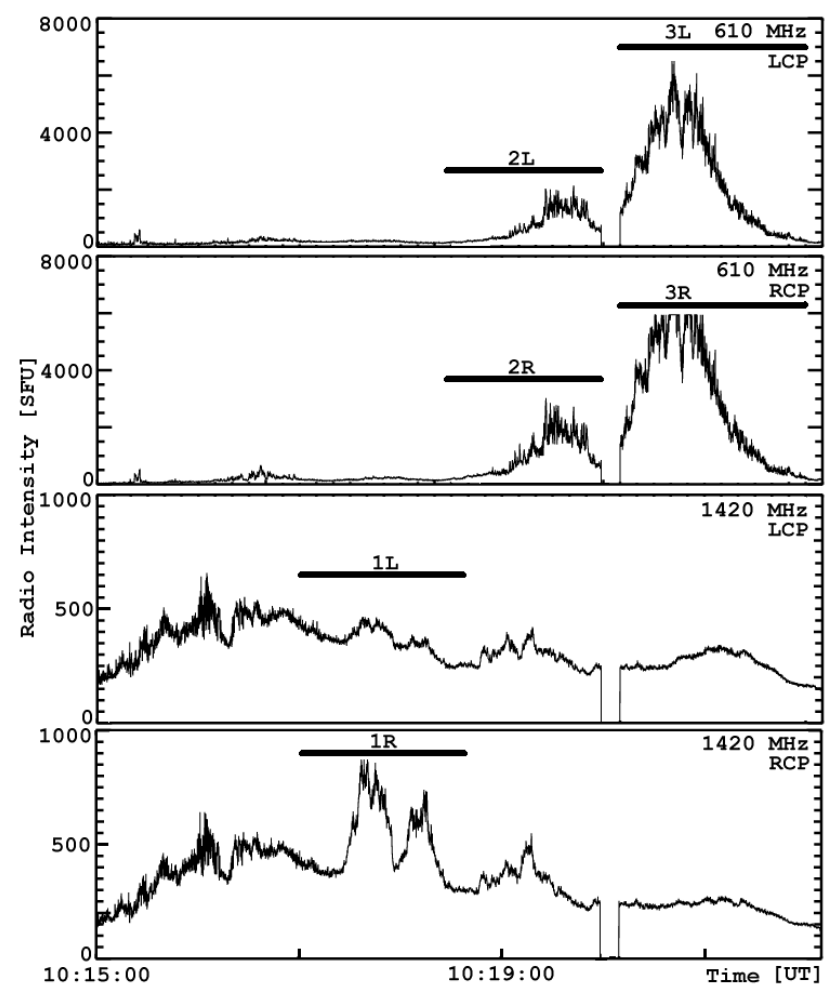

Fig. 7. Left (L) and right (R) circular polarization of the 12 April 2001 fluxes observed at 1420 and $610 \mathrm{MHz}$ by the Trieste radiopolarimeter. The intervals studied statistically are designated by numbers 1,2 , and 3 .

tween neighboring plasmoids, penetrate into the plasmoids, and along their trajectories generate individual fast drift bursts in specific slowly drifting structures. A series of drifting structures thus maps the reconnection process. Their negative drifts correspond to motions of the plasmoids along the flare current sheet upwards in the solar atmosphere. This drift varies, expressing the variations of the reconnection rate. After some maxima (reconnection rate), the frequency drift and thus the reconnection rate decrease at the ending phase of the series. Using this model we can interpret the time behavior of the interacting drifting structures at 13:07:52 UT13:09:00 UT (Fig. 3) as radio manifestation of the coalescence of neighboring secondary plasmoids. For more details, see paper by Karlický (2004).

\subsection{The 12 April 2001 event}

On 12 April 2001 the GOES satellite observed a soft X-ray event which started at 09:39 UT, with maximum at 10:28 UT and end at 10:49 UT. This event was classified as X2.0 and localized in the active region NOAA AR 9415.

Figure 6 shows the radio spectrum of this flare in a broad range of frequencies from 40 to $4500 \mathrm{MHz}$ (40-800 MHz observed by Potsdam and $800-4500 \mathrm{MHz}$ by Ondřejov radiospectrographs). In radio waves the flare started at 10:14:30 UT at about $1500 \mathrm{MHz}$ with a series of fast drifting bursts covering the band from 200 to at least $4500 \mathrm{MHz}$. 
Below $800 \mathrm{MHz}$ these bursts formed a precursor which was followed by the type II burst at 10:17 UT-10:33 UT in the metric range (Klassen et al., 2003).

Then during 10:16:00 UT-10:17:30 UT and 10:17:20 UT10:22:00 UT in the range 450-1000 $\mathrm{MHz}$ and 450-1500 $\mathrm{MHz}$ respectively, high-frequency slowly drifting pulsating structures were observed. The second one was stronger and its global frequency drift was about $-1.8 \mathrm{MHz} \mathrm{s}^{-1}$.

The radio emission of the flare was recorded also on two single frequencies $(610$ and $1420 \mathrm{MHz}$ ) by the Trieste radiopolarimeter with $1 \mathrm{~ms}$ time resolution (Fig. 7). While the beginning part of the flare was practically unpolarized, at times when the single frequency observations cut the second, i.e. stronger slowly drifting structure (10:17:20 UT10:18:30 UT on $1420 \mathrm{MHz}$ and 10:18:30 UT-10:22:00 UT on $610 \mathrm{MHz}$ ) the radio emission was polarized in the rightcircular sense. The mean polarization at these times was 50 $60 \%$ on $1420 \mathrm{MHz}$ and $10-20 \%$ on $610 \mathrm{MHz}$, respectively.

For the statistical analysis three intervals cutting the stronger slowly drifting structure on 1420 and $610 \mathrm{MHz}$ were selected (Fig. 7, intervals designated 1R, 1L, 2R, 2L, 3R, $3 \mathrm{~L} ; \mathrm{R}$ and $\mathrm{L}$ mean right and left circular polarization, respectively). (Remark: the receiver at $610 \mathrm{MHz}$ was saturated when the flux density reached the level about 6000 SFU, that is more evident in the R-channel.) We used the Fourier method. Power spectra for the selected intervals are shown in Fig. 8. (After the subtraction of the spectrum obtained during the pre-flare interval.) We recognized periods in the range of seconds (0.9-7.5 s) with high statistical probability (86-95\%); they are summarized in Table 1. Going to shorter periods, first many individual periods in the range of $\sim 0.2$ $0.9 \mathrm{~s}$ can be seen and then in the range of $0.2-0.06 \mathrm{~s}$ Fourier spectra show power-law form $\left(\sim \mathrm{f}^{-\gamma}\right)$ with power-law index $\gamma$ in the 1.3-1.6 range (see Table 1). For even shorter periods $(<0.06 \mathrm{~s})$ only noise was present (Fig. 8).

Moreover, the data in the intervals $1 \mathrm{R}, 1 \mathrm{~L}, 2 \mathrm{R}, 2 \mathrm{~L}$ and in the non-saturated parts of the intervals $3 R$ and $3 L$ were tested with respect to low-dimensional determinism and nonlinearity (surrogate data tests and correlation dimensions, for the methods see Mészárosová et al., 2003). Non-linear behavior was found in the non-saturated parts of the $3 \mathrm{R}$ and $3 \mathrm{~L}$ intervals. All intervals indicated high dimensional or stochastic processes.

\subsubsection{Time scales interpretation}

Based on the paper by Kliem et al. (2000) we propose that during the turbulent reconnection local enhancements of the electric field are generated and simultaneously electron beams are accelerated. These beams generate pulses inside slowly drifting pulsating structures through bump-on-tail instability and wave transformation. The pulses are spatially localized (i.e. localized also in the radiation frequency) due to trapping of beams in the O-type magnetic field structures (plasmoids). The partial right-circular polarization of the high-frequency structure speaks in favour of radio emission at the fundamental plasma frequency.
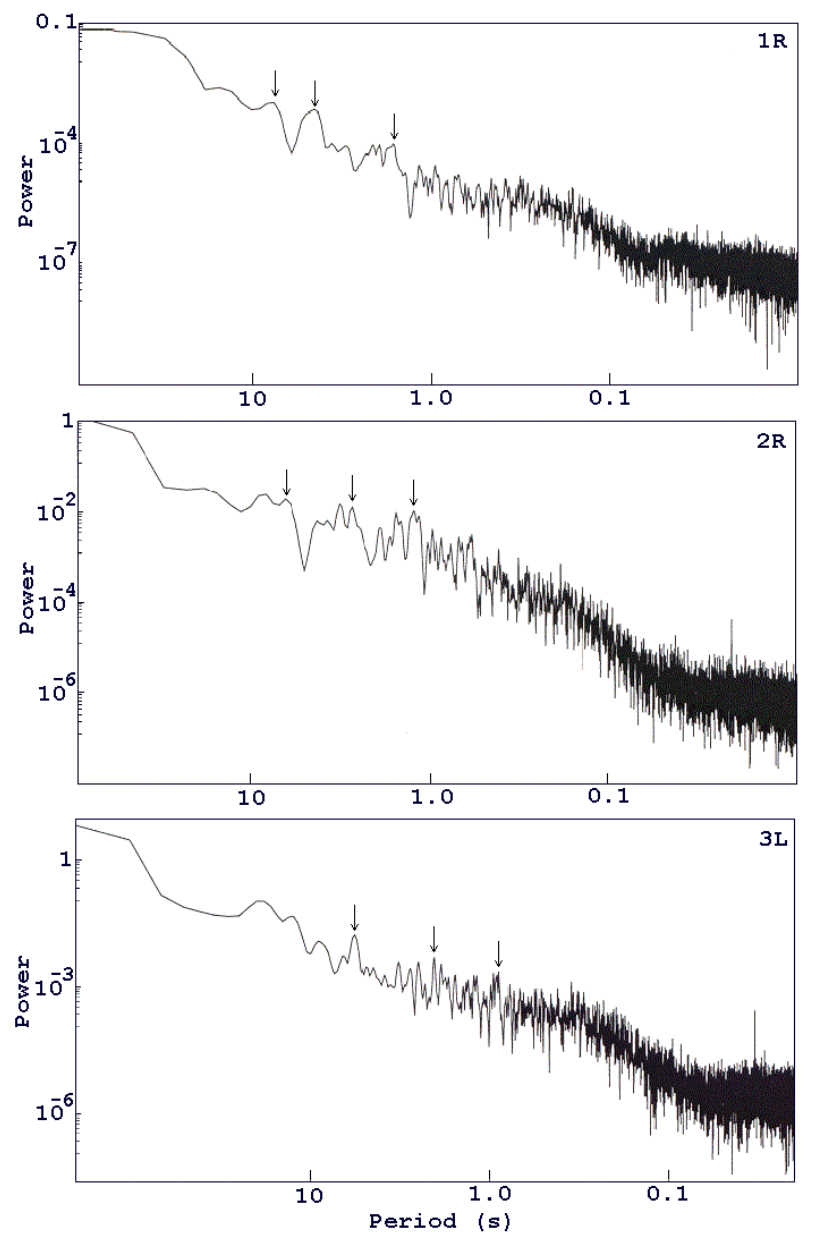

Fig. 8. Fourier power spectra in three selected intervals $1 \mathrm{R}, 2 \mathrm{R}$, $3 \mathrm{~L}$ during the drifting structures (see Fig. 7). Fourier power spectra made in the interval before the bursts (radio flux of "quiet sun") were subtracted. The arrows show the periods reported in Table 1.

In such a scenario we propose that spatial scales of plasmoids in the current sheet correspond to those generated by a cascade of tearing instabilities - multi-scale reconnection (Shibata and Tanuma, 2001). On the other hand, time scales of enhanced electric fields, localized between neighbouring plasmoids, follow from their subsequent coalescence processes as described by Tajima et al. (1987).

\subsubsection{MHD model of reconnection}

In the following we simulate the proposed processes and then we compare them with observations. In the model we solve the following set of equations

$$
\begin{array}{r}
\frac{\partial \rho}{\partial t}+\nabla \cdot(\rho \boldsymbol{u})=0 \\
\rho \frac{\partial \boldsymbol{u}}{\partial t}+\rho(\boldsymbol{u} \cdot \nabla) \boldsymbol{u}=-\nabla p+\boldsymbol{j} \times \boldsymbol{B} \\
\frac{\partial \boldsymbol{B}}{\partial t}=\nabla \times(\boldsymbol{u} \times \boldsymbol{B})-\nabla \times(\eta \boldsymbol{j}) \\
\frac{\partial U}{\partial t}+\nabla \cdot \boldsymbol{S}=0,
\end{array}
$$


Table 1. Frequencies, analyzed intervals, periods and power-law indices. For periods, see the arrows in Fig. 8.

\begin{tabular}{cccc}
\hline Interval (see Fig. 7) & $1 \mathrm{R}$ & $2 \mathrm{R}$ & $3 \mathrm{~L}$ \\
\hline Frequency (MHz) & 1420 & 610 & 610 \\
Time Interval & $10: 17: 10 \mathrm{UT}-10: 18: 40 \mathrm{UT}$ & $10: 18: 30$ UT-10:19:59 UT & $10: 20: 10$ UT-10:22:00 UT \\
$\begin{array}{c}\text { Periods (s) } \\
\text { Probabilities } \\
\text { of Periods (\%) }\end{array}$ & $7.5,4.5,1.6$ & $6.3,2.7,1.2$ & $5.7,2.0,0.9$ \\
$\begin{array}{c}\text { Power-law Index } \gamma \\
\text { of Fourier spectrum } \\
\text { in Period Range (s) }\end{array}$ & 1.4 & $95,94,95$ & $86,87,89$ \\
\hline
\end{tabular}
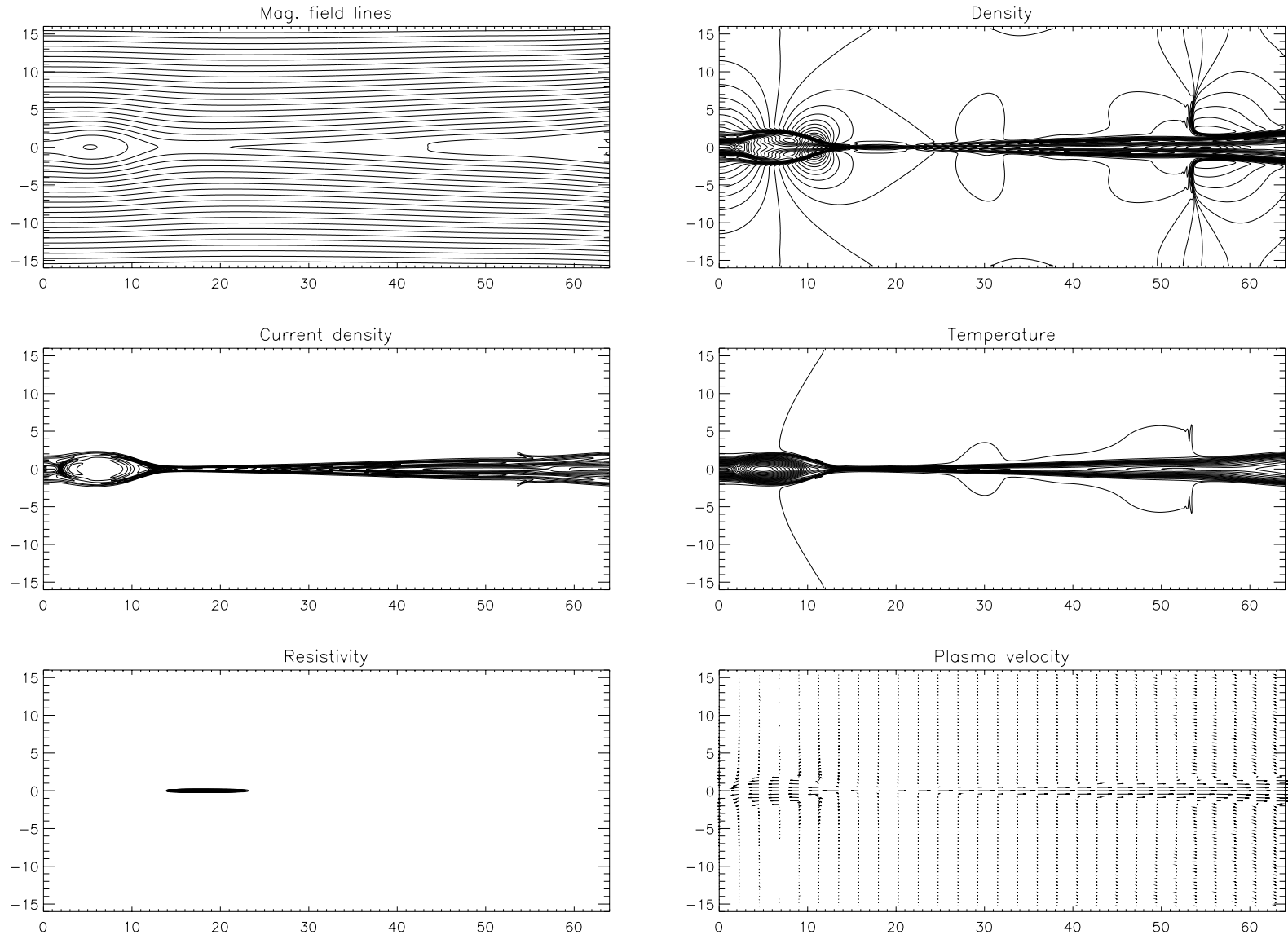

Fig. 9. Magnetic filed lines, current density, places of enhanced electric resistivity, plasma density and temperature isolines, and velocity field during the model reconnection process at $280 \tau_{A}$.

where $\rho$ is the plasma density, $\boldsymbol{u}$ is the plasma velocity, $p$ is the plasma pressure, $\boldsymbol{j}$ is the current density, $\boldsymbol{B}$ is the magnetic field, $U$ is the total energy density, and $S$ the energy flux (see for details, Kliem et al., 2000).

This system is then numerically solved using the 2D LaxWendroff scheme inside a rectangular box with symmetric boundary conditions on $x$ and $y$ axes and with free outer boundary. The Harris current sheet perturbed for finite time by anomalous resistivity is used at the initial state. See papers by Karlický (1988) and Kliem et al. (2000) for details.
Computations are made for 1200 Alfvén transit time $\tau_{A}$ and the results are shown in Figs. 9 and 10. Figure 9 presents the state of the magnetic field reconnection (magnetic field lines, current density, electric resistivity, plasma density and temperature isolines, and plasma velocity field) at $t=280 \tau_{A}$. In Fig. 9 one magnetic island (plasmoid) and reconnection plasma outflow can be seen in the current sheet at positions $x \sim 5, x=20-64$, respectively. The energy release is concentrated in regions of high resistivity (position $x=13-23$ ). 

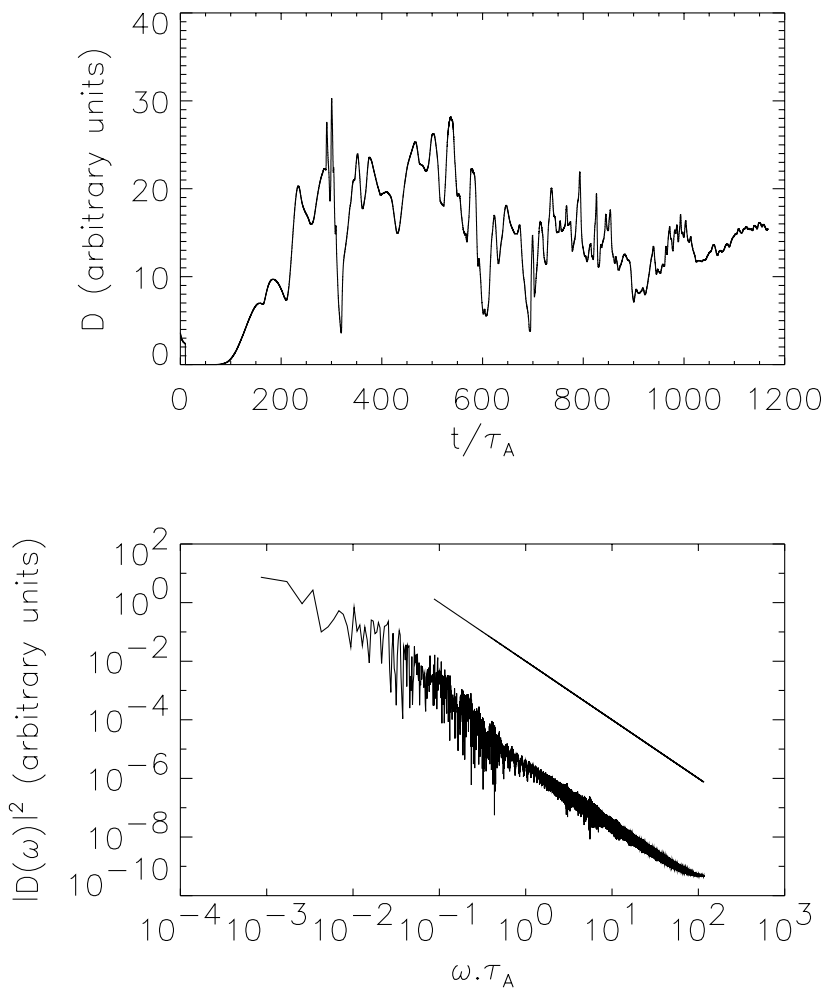

Fig. 10. Time variations of the dissipated power $D$ computed by the 2-D MHD numerical model and their Fourier power spectrum. $\tau_{A}$ is the Alfvén transit time. The line corresponding to the power-law spectrum with power-law index 2 is added for comparison.

\subsubsection{Comparison with radio flux variations}

In order to compare directly the dynamical characteristics of the modeled reconnection process with those obtained from the observed radio data the temporal evolution of the integral dissipated power

$D=\int_{\text {Box }} \eta(x, y) j^{2}(x, y) \mathrm{d} x \mathrm{~d} y$

is computed (Fig. 10). It shows that the reconnection process is strongly variable (intermittent, bursting) in time.

Then, we made the Fourier power spectrum of these variations (Fig. 10). As can be seen it has a power-law form with power-law index 2 .

\section{Radio bursts generated in reconnection outflows}

According to the reconnection theory the intrinsic part of the reconnection process is the plasma outflow where the MHD turbulence is probably generated (Chiueh and Zweibel, 1987). Moreover, due to acceleration processes, particle beams and other types of non-equilibrium particle distribution functions unstable with respect to growth of various kinds of (electrostatic) plasma waves are expected. These conditions may produce high level of electrostatic plasma waves as well as (due to turbulent environment, see Benz and

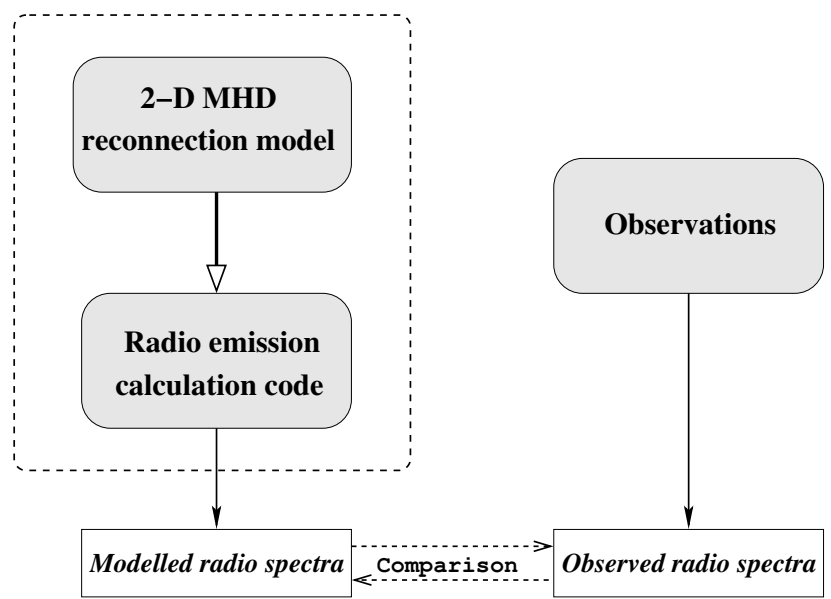

Fig. 11. Schema of the comparison between the two types of spectra.

Wentzel, 1981) their effective conversion to electromagnetic mode. As result, one may expect a significant radio radiation from the reconnection with features typical for plasma emission from turbulent sources.

\subsection{Observational evidence for turbulence in solar flare ra- dio sources}

There are several types of radio bursts identified in the decimetric range indicating that their sources are located in turbulent environment. This can be documented by their dynamical characteristics and/or distribution of spatial scales in the source inferred from observations. In particular, two of this class will be dealt with in this paper:

\subsubsection{Lace bursts}

In the radio spectrum the lace burst consists of very narrowband (about $50 \mathrm{MHz}$ ) lines rapidly varying in frequency and intensity. Instantaneous line profile is asymmetric showing a rather sharp high-frequency edge and gradual decay in the low-frequency part (wing). Emission lines are mutually superimposed forming thus a lace burst. The frequencies and intensities change in fractions of a second. For further details about the lace burst characteristics, see Karlický et al. (2001). Analyzing temporal evolution of the well defined high-frequency boundary of the emission line it was found, that the Fourier power spectra show power-law profiles with indices close to 2 . Such self-similar dynamics indicates the presence of so called inertial range of scales in turbulent source.

\subsubsection{Decimetric spikes}

The global appearance of the dm-spikes in dynamic spectra resembles a cloud of very narrow-band and short-duration bright bursts. It was found, that adjacent spikes in the cloud are correlated to some extent. For details about spectral properties of dm-spikes see for example the review by Benz 

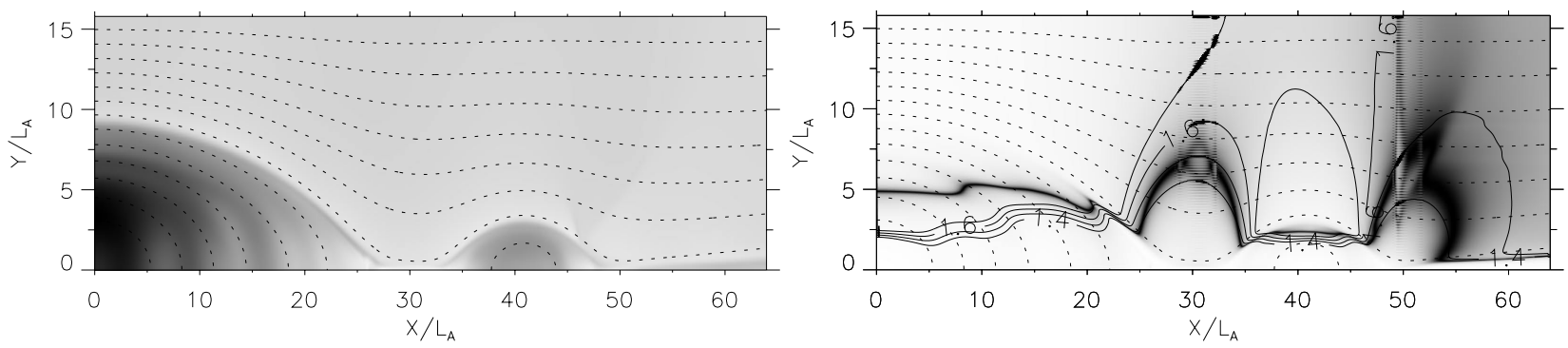

Fig. 12. Snapshot of the reconnection dynamics in the first quadrant at the time $t=900 \tau_{\mathrm{A}}$. Left: Magnetic field (dashed lines) and density (gray levels) structures. The density fluctuations and the secondary plasmoid formation in the reconnection are clearly visible. Right: Locations of sources of the radio emission (gray-level thin area) inside the reconnection. The solid contours mark out iso-surfaces of the constant radiated frequencies 1.4, 1.5, 1.6, and 1.7 GHz. Dashed ones are magnetic field lines (cf. the left part).

(1986). In the studies by Karlický et al. (1996, 2000) and Zlobec and Karlický (1998) Fourier analysis was applied to distribution of spatial scales inside the source inferred from the frequency bandwidths. Authors found power-law spectra with power-law indices in the range of $0.80-2.85$. This spatial scaling indicates again the presence of turbulence in the $\mathrm{dm}$-spikes sources.

\subsection{The model}

In the present paper, as an extension of our previous ideas (Karlický et al., 2001; Bárta and Karlický, 2001, 2003), we propose and numerically test the following hypothesis: the lace and the dm-spikes bursts are generated inside turbulent reconnection flows; the radiation is caused by some kind of plasma emission process. The instability of upper-hybrid (UH) waves due to an excess of transversal electron temperature is considered here in agreement with the concept of betatron acceleration in the collapsing magnetic trap suggested recently by Karlický and Kosugi (2004).

\subsubsection{Model overview}

To test our hypothesis the following scheme is used (see Fig. 11): First, a set of MHD equations (1) is solved in two dimensions for the initial Harris current sheet (see Sect. 2.2.2). Plasma parameters (plasma and energy densities, magnetic field, and velocity) are computed in successively growing times inside reconnection region. Density and magnetic field structures are then used as an input for calculation of the double-resonance radio emission. Final results - artificial radio spectra - can be directly compared with observations.

\subsubsection{Radio emission calculation}

As already mentioned, radio emission under study is considered to be due to double-resonance instability of the UH waves (Zheleznyakov and Zlotnik, 1975) and their subsequent transformation into the escaping electromagnetic mode. The relevant growth rate in the considered case of equilibrium electron distribution function perturbed by the
bi-Maxwellian beam with $T_{\perp}>T_{\|}$and relative density $\alpha$ is (Mikhailovski, 1975)

$$
\begin{array}{r}
\gamma(\boldsymbol{k})=-\frac{\sqrt{\pi} \alpha \omega^{3}(\boldsymbol{k})}{v_{T e \|}^{3} k^{2}\left|k_{\|}\right|} \sum_{s=-\infty}^{\infty}\left\{I_{s} \exp \left(-z_{\perp}\right) \times\right. \\
\left.\exp \left(-\frac{\Delta \omega^{2}}{k_{\|}^{2} v_{T e \|}^{2}}\right)\left(\Delta \omega+s \omega_{\mathrm{Be}} \frac{T_{\|}}{T_{\perp}}\right)\right\},
\end{array}
$$

where the frequency mismatch

$\Delta \omega \equiv \omega(\boldsymbol{k})-k_{\|} v_{\|}-s \omega_{\mathrm{Be}}$

and the real part of the dispersion equation (in the limit $k_{\|} \ll k_{\perp}$ where the growth rate has a maximum) can be approximated as

$\omega(\boldsymbol{k})=\sqrt{\omega_{\mathrm{UH}}^{2}-\frac{\omega_{\mathrm{Be}}^{2} \omega_{\mathrm{pe}}^{2}}{\omega_{\mathrm{UH}}^{2}} \frac{k_{\|}}{k^{2}}}$.

Density and magnetic field structures evolving during the reconnection process govern the radio emission by means of frequencies $\omega_{\mathrm{pe}}$ and $\omega_{\mathrm{Be}}$ contained implicitly in relation (2). For detailed explanation of other used (and not important here) quantities we refer to Mikhailovski (1975) and Bárta and Karlický (2001).

Dynamics of the UH mode is then described by

$E(\boldsymbol{k}, t+\Delta t)=E(\boldsymbol{k}, t) \cdot \exp [(\gamma(\boldsymbol{k})-v) \cdot \Delta t]$

in each point of the source with effects of instability saturation taken into account. Further, the instability is assumed to be suppressed completely if the temporal scale of variations of local plasma parameters approaches the characteristic growth time of the UH waves.

Mode conversion into the observable radio waves is consistently supposed to be due to the scattering of the UH waves on the low-frequency plasma inhomogeneities in the radio source and it is not calculated here. In order to describe qualitatively the spectral dynamics of radio emission the proportionality between radio flux and energy density of UH waves at respective frequencies was assumed instead. 


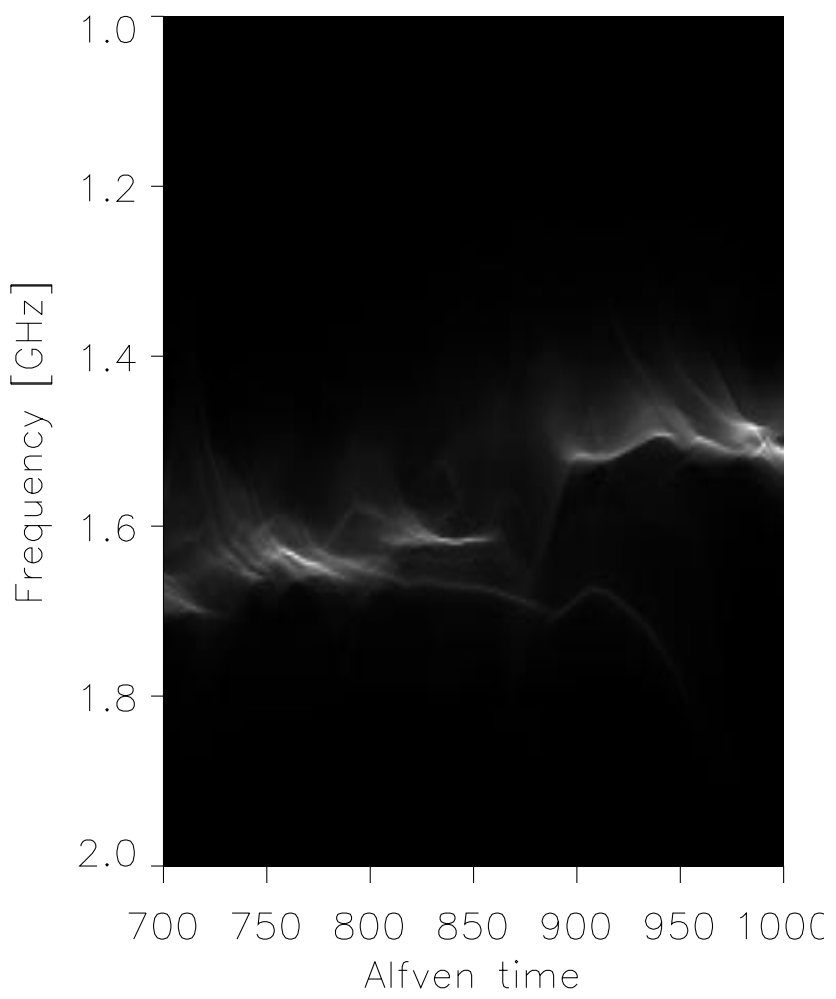

Fig. 13. Dynamical spectrum of the modeled radio emission from reconnection. Scaling factors used for magnetic field and electron density (see Kliem et al., 2000) are $B_{0}=250 \mathrm{G}$ and $n_{0}=$ $3 \times 10^{10} \mathrm{~cm}^{-3}$, respectively. The emission was considered at the third harmonic $(s=3)$.

\subsection{Results}

An example of the MHD reconnection modeling is shown in the left part of Fig. 12 (see also Fig. 9). The obtained $\boldsymbol{B}$-field and the density structures are then passed into the radio emission calculation code. However, besides the above quantities the growth rate (2) depends also on the parameters and the spatial structure of the perturbed distribution function which cannot be derived from the MHD simulation. Therefore two - in some sense extreme - cases were studied:

\subsubsection{Wide-spread unstable distribution function}

In this approach the anisotropic beam perturbing equilibrium distribution function is assumed to fill homogeneously the whole reconnection box. Obtained results are shown in Figs. 12 (right part), 13, and 14. Figure 13 shows the dynamic spectrum of the modeled radio emission due to reconnection. It qualitatively reproduces the features seen on the spectra of lace bursts - slowly varying changes of the emission line position and, also line profile with sharp cutoff at the high-frequency end as well as a diffusive low-frequency part. This is pronounced even more clearly in the instantaneous spectrum taken at time $t=900 \tau_{\mathrm{A}}$ (Alfvén time) (Fig. 14).

The right part of Fig. 12 shows the localization of the radio emission sources at the same time. They are confined

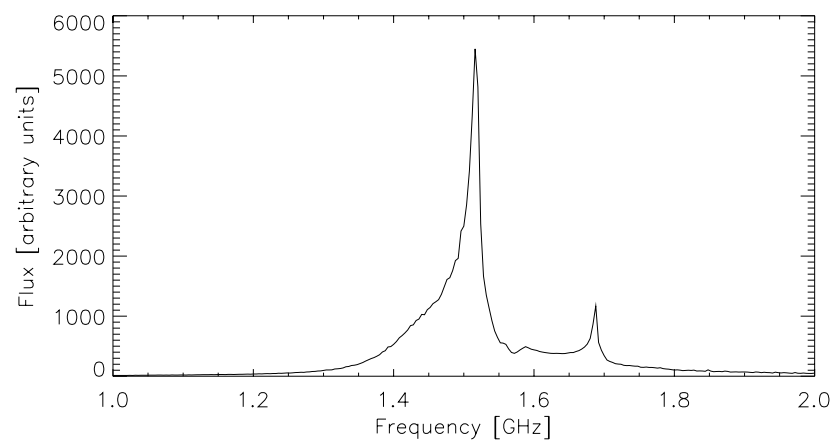

Fig. 14. Modeled instantaneous spectrum of radio emission from reconnection at time $t=900 \tau_{\mathrm{A}}$. The sharp high-frequency boundary and the diffusive low-frequency part are clearly visible.

in the thin region around the surface fulfilling the resonant condition

$\Delta \omega \approx \omega_{\mathrm{UH}}-s \omega_{\mathrm{Be}}=0$

inferred from the Eqs. (2), (3), and (4). The solid contours show the radiated frequency isolines. In addition, the magnetic field lines are plotted (dashed curves) to gain a better image of the source position within the reconnection process.

As can be seen in Fig. 12, even several surfaces of constant radiated frequency cross the area of emission localization. It appears quite surprising in this context, that the resulting emission is not broad-band, but with well defined peak at $\approx 1.5 \mathrm{GHz}$ (see Fig. 14). The explanation is schematically sketched in Fig. 15. Let $B W$ is the half-bandwidth of one frequency channel of a radio receiver centered around the angular frequency $\omega_{r}$. Then the outlined belt around the isosurface $\omega_{r}=$ const. represents the volume that potentially contributes to radiation in this channel. On the other hand, let $R W$ is the characteristic frequency half-width of the doubleresonance. Then, significant contributions to emission come only from the belt around the surface, where resonant condition (6) is fulfilled. The radio flux $F$ in the given channel can be thus estimated as some factor times the cross-section of both belts:

$$
F \propto \frac{B W \cdot R W}{\left|\nabla \Delta \omega \times \nabla \omega_{r}\right|} .
$$

This relation ensures that the principal contribution to the radio flux comes from the (rare) regions where the resonant surface and the surface of constant radiated frequency are locally parallel.

\subsubsection{Confined non-Maxwellian perturbation}

As an alternative to the assumption of unstable distribution function homogeneously spread over the whole reconnection region we studied the non-Maxwellian perturbation confined in one thin flux-tube carried away by the reconnection flow. This approach extends our previous work (Bárta and Karlický, 2003) by inclusion of dynamics of the radio source inside the reconnection. 


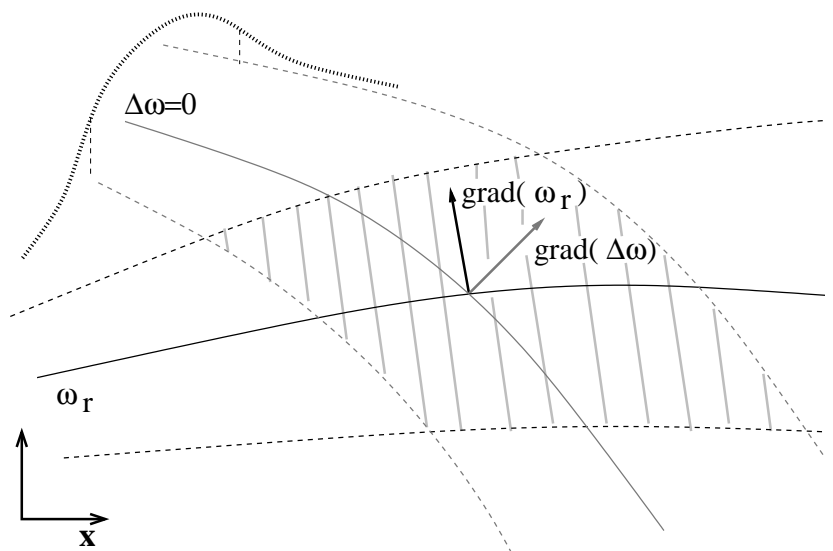

Fig. 15. Outline of contributions to the radio flux at a given frequency channel centered around angular frequency $\omega_{r}$ from various places in the reconnection box. Maximum contributions come from points where the resonant surface and the surface of radiated frequency are locally parallel.

To simulate the presence of small scales in the MHD turbulence which are, unfortunately, smoothed out in the present MHD reconnection model we perturbed the computed density and the magnetic field profiles in the studied 1-D source (along one thin flux tube) by artificial high frequency turbulent variations. Extending the spectrum of superimposed perturbations to short scales, the qualitative transition was found to be caused by an interruption of the instability of the $\mathrm{UH}$ waves by too fast changes of background parameters. Results are shown in Fig. 16 and the spectrum was identified with one chain of decimetric spikes. Clouds of spikes observed more frequently can be reproduced as superposition of several chains - for example as consequence of several discrete flux-tubes filled by perturbed distribution function see Bárta and Karlický (2001). On the other hand, in the stationary atmosphere the same radiation mechanism leads to zebra bursts (see e.g. Sawant et al., 2002).

To test the presented hypothesis further also profiles of individual spikes were studied in addition to the global spectral dynamics. As was pointed out by Güdel and Benz (1990) the time profiles of individual spikes exhibit Gaussian raise and main phases followed by exponential decay. To prove that, authors used the logarithmic derivative $(L D F)$ of the radio flux $F$ reduced of background continuum

$$
L D F=\frac{\mathrm{d}}{\mathrm{d} t} \log (F(t))=\frac{1}{F} \cdot \frac{\mathrm{d} F}{\mathrm{~d} t} .
$$

Prevailing exponential decay phase is indicated by a constant value of $L D F$. On the other hand, a linear decrease of the logarithmic derivative corresponds to the Gaussian time profile of radio flux. Analyzing individual spikes computed using our model the same characteristics were found also for modeled spectra. Comparison of results of the LDF analysis applied to the observed and the modeled spikes is shown in Fig. 17.

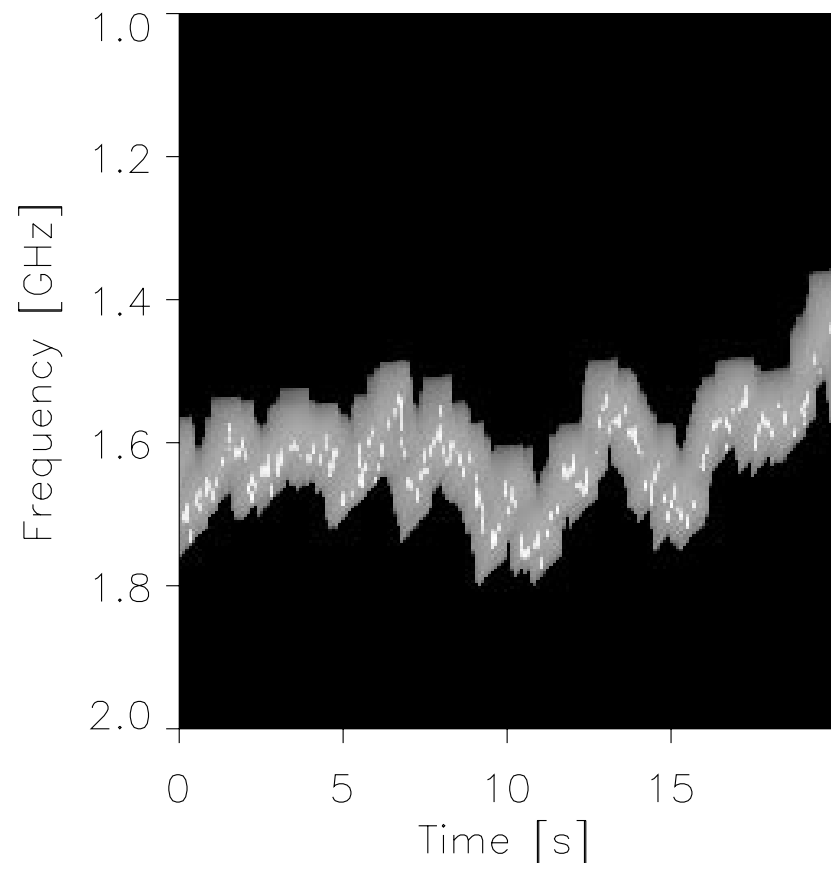

Fig. 16. The 1.0-2.0 GHz modeled radio spectrum of a chain of spikes. The radio flux shown is in a logarithmic scale. MHD scaling parameters are $B_{0}=200 \mathrm{G}, n_{0}=3 \times 10^{10} \mathrm{~cm}^{-3}$, and $s=4$.

\section{Radio emission of the reconnection outflow termina- tion shocks}

It is commonly accepted that below the rising filament a current sheet is formed in which the reconnection takes place (Fig. 4). It is assumed that while plasma reconnection inflows are in a horizontal direction, the plasma reconnection outflows are in vertical direction. It is believed that these reconnection outflows interact with the above-lying plasmoid and the under-lying flare loops. Here so the called termination shocks can be formed. A question arises if such termination shocks can be manifested in the radio spectra in a way similar to flare shocks (type II radio bursts). In the paper by Aurass et al. (2002) a unique example of decimetric type IIlike herringbone structure was shown (see Fig. 18). It was proposed that this radio emission can be considered as the radio emission from the termination shock.

\section{$5 \mathrm{H} \alpha$ of the 18 September 1995 eruptive prominence}

In the paper by Kotrč et al. (1998) a detailed analysis of the September 18, 1995 eruptive prominence was presented. Using a combined soft X-ray SXT/Yohkoh, $\mathrm{H} \alpha$ image and spectral observations it was found that the $\mathrm{H} \alpha$ spectra from the location of the loop-loop interaction are split into two components. The derived Doppler velocities are in the -100 $+50 \mathrm{~km} \mathrm{~s}^{-1}$ range. This spectral splitting indicates the bidirectional plasma flow as expected in the reconnection process. 
a)
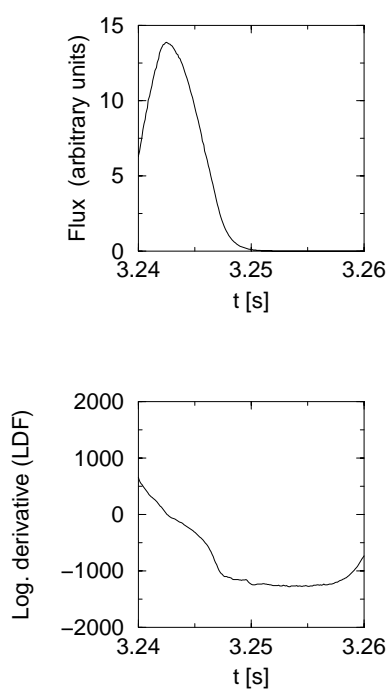

b)
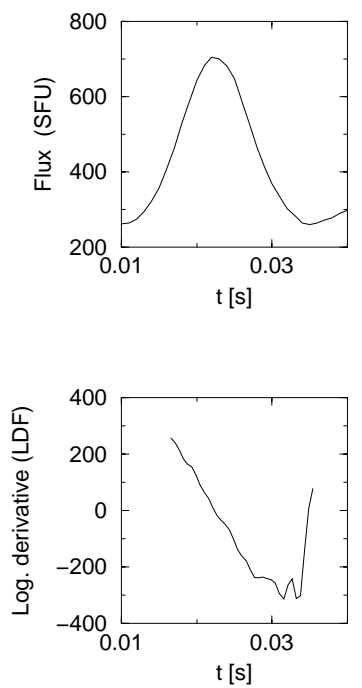

Fig. 17. Comparison between modeled and observed spike profiles using the logarithmic derivative of the flux $(L D F)$. (a) time profile at $1670 \mathrm{MHz}$ and its logarithmic derivative of a modeled spike. The beginning of the spike profile is not included due to high values of numerical noise in $L D F$. (b) time profile and its $L D F$ of a spike recorded at the Trieste Observatory, Italy at $1420 \mathrm{MHz}$. The background of $215 \mathrm{SFU}$ was subtracted before the analysis.

\section{Conclusions}

New radio and $\mathrm{H} \alpha$ diagnostics of the solar flare reconnection process are presented.

Is is proposed that a series of high-frequency slowly drifting structures manifest plasmoids formed in the extended current sheets and thus these structures map the magnetic field reconnection. An increase of their frequency drifts corresponds to an increase of the reconnection rate.

Series of pulses in the drifting pulsating structures are explained by a series of electron beams accelerated in the electric field generated during the tearing and the coalescence processes in the bursting (multi-scale) reconnection. Thus, Fourier spectra of time variations of the drifting pulsating structures can be used for diagnostics of acceleration processes in the magnetic field reconnection.

Using 2-D MHD modeling it was shown that Fourier spectrum of time variations of the dissipative power has a powerlaw form with power-law index 2. This result is an extension of the results of Valdivia et al. (2003), who made similar computations, but in the 1-D model only. They obtained Fourier spectrum with power-law index 1 . Thus, we expect that in reality, i.e. in 3-D case, Fourier power spectrum has also power-law form, but with power-law index $\geq 2$. These results show that tearing and coalescence processes in the multi-scale reconnection are self-similar, i.e. they have not any characteristic scale length.

Ryabov et al. (1997) studied fine structures in 2.5 and 2.85 $\mathrm{GHz}$ radio bursts and they found power-law Fourier spec-

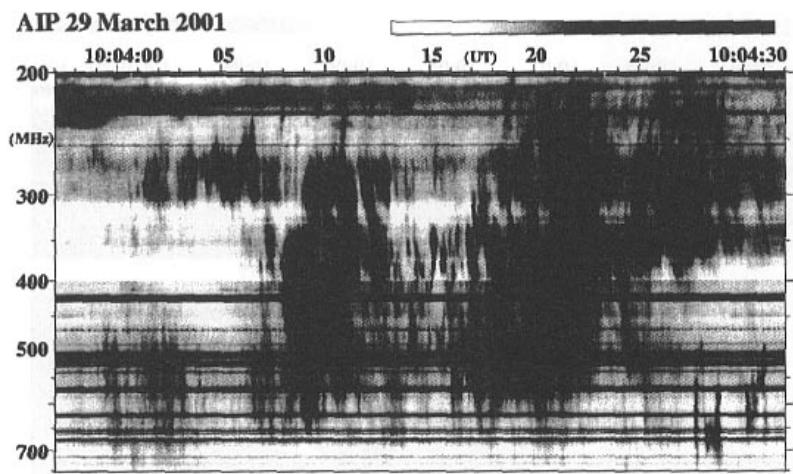

Fig. 18. Decimetric type II-like herringbone structure.

tra with power-law index in the range $1-2$, which is similar to our case (1.3-1.6). They suggested a model of uncorrelated pulses as capable to generate such spectra. We think that the proposed multi-scale reconnection processes belong to the same class of processes. On the other hand, deviations from power-law dependence can be expected for longer time scales (corresponding to larger spatial scales) due to the limited volume of the flare. For longer period range we really found well specified periods of 0.9-7.5 s (Table 1).

We think that the difference in the power-law indices of Fourier spectra of the observed and of the simulated data are due to non-linear processes, which in reality connect the dissipation power in the reconnection and the radio flux output. On the other hand, this difference gives us a limit for conversion processes (dissipation power $\rightarrow$ electron acceleration $\rightarrow$ plasma wave generation $\rightarrow$ transformation of plasma waves into electromagnetic ones $\rightarrow$ radiative transfer of generated radio waves).

The acceptance of the presented interpretation of the lace and the spike bursts would have some applicable consequences. If true, it represents the first possibility of direct detection and analysis of one of key parts of the reconnection process. Particularly, the turbulence in reconnection outflows can be studied using the Fourier analysis of temporal variations of the emission line position during the lace burst. Such an analysis was done in the paper by Karlický et al. (2001). First, obtained spectra need to be converted from temporal to spatial scale by some way. Since the temporal variations imply basically from a transit of spatial inhomogeneities through the radio source one can estimate transformation between spatial and temporal scales as

$\Delta l \approx v_{\mathrm{A}} \Delta t$.

If this re-calculated power spectrum contains significant breakpoints well known from the turbulence theory (see e.g. Kurien and Sreenivasan, 2000) the following important scales of the reconnection flow dynamics can be estimated: 1) Energy input scale. If found in observed spectra it would give us an estimation of the transversal dimensions of outflow.

2) Dissipation scale. It contains a combination of the energy dissipation rate and the electric resistivity. Estimating one of 
these parameters independently, the second one can be calculated, if this scale can be identified in the observed spectrum.

Furthermore, in the prevailing case of high gradient of magnetic field (comparing with density gradient) along the radio source the frequency changes are mainly due to density variations. Hence, $\left\langle\delta n^{2}\right\rangle$ can be easily computed integrating the observed spectrum of frequency variations re-calculated firstly to density changes over its domain.

Finally, there are indications that the radio plasma emission from the reconnection termination shock and also $\mathrm{H} \alpha$ spectroscopy can give us additional information about the reconnection process.

Acknowledgements. This work was supported by the grant A3003202 of the Academy of Sciences of the Czech Republic. The authors thank two anonymous referees for helpful comments and suggestions.

Edited by: A. Chian

Reviewed by: two referees

\section{References}

Asai, A., Yokoyama, T., Shimojo, M. and Shibata, K.: Downflow motions associated with impulsive nonthermal emissions observed in the 23 July 2002 solar flare, ApJL, 605, L77-L80, 2004.

Aurass, H., Karlický, M., Thompson, B.J. and Vršnak, B.: Radio shocks from reconnection outflow jet?-New observation, in Multi-wavelength observations of coronal structure and dynamics, edited by Martens, P. C. H., and Cauffman, D. P.), COSPAR Coll. Ser., 13, 401-404, 2002.

Bárta, M. and Karlický, M.: Turbulent plasma model of the narrowband dm-spikes, A\&A 379, 1045-1051, 2001.

Bárta, M. and Karlický, M.: Radio manifestation of reconnection outflow jets, in Solar Variability as an Input to the Earth Environment, edited by Wilson, A., ESA-SP 535, 471-476, 2003.

Benz, A. O.: Millisecond radio spikes, Sol. Phys. 104, 99-110, 1986.

Benz, A. O. and Wentzel, D. G.: Coronal evolution and solar type I radio bursts - an ion-acoustic wave model, A\&A, 94, 100-108, 1981.

Chiueh, T. and Zweibel, E.: The structure and dissipation of forced current sheets in the solar atmosphere, ApJ, 317, 900-917, 1987.

Curdt, W., Innes, D. E., and Wilhelm, K.: SUMER observations of bi-directional flows in coronal plasmas, Proc. ESA SP-421, 123-128, 1998.

Fárník, F., Garcia, H., and Karlický, M.: New solar broad-band hard X-ray spectrometer: First results, Solar Phys., 201, 357372, 2001.

Güdel, M. and Benz, A. O.: Time profiles of solar radio spikes, A\&A, 231, 202-212, 1990.

Hudson, H. S., Kosugi, T., Nitta, N., and Shimojo, M.: Hard Xradiation from a fast coronal ejection, ApJ, 561, L211-L214, 2001.

Jiřička, K., Karlický, M., Kepka, O., and Tlamicha, A.: Fast drift burst observations with the new Ondřejov radiospectrograph, Solar Phys., 147, 203-206, 1993.
Karlický, M.: Response of the current sheet to a time-limited enhancement of electrical resistivity, Bull. Astron. Czechosl. 39, 13-23, 1988.

Karlický, M.: Series of high-frequency slowly drifting structures mapping the flare magnetic field reconnection, A\&A, 417, 325332, 2004.

Karlický, M. and Odstrčil, D.: The generation of MHD shock waves during the impulsive phase of the 27 February 1992 flare, Solar Phys., 155, 171-184, 1994.

Karlický, M. and Kosugi, T.: Acceleration and heating processes in a collapsing magnetic trap, A\&A, 419, 1159-1168, 2004.

Karlický, M., Sobotka, M., and Jiřička, K.: Narrowband dm-spikes in the $2 \mathrm{GHz}$ frequency range and MHD cascading waves in reconnection outflows, Sol. Phys., 168, 375-383, 1996.

Karlický, M., Jiřička, K., and Sobotka, M.: Power-law spectra of 12 GHz narrowband dm-spikes, Sol. Phys. 195, 165-174, 2000.

Karlický, M., Bárta, M., Jiřička, K., Mészárosová, H., Sawant, H. S., Fernandes, F. C. R., and Cecatto, J. R.: Radio bursts with rapid frequency variations - Lace bursts, A\&A 375, 638-642, 2001.

Karlický, M., Fárník, F., and Mészárosová, H.: High-frequency slowly drifting structures in solar flares, A\&A, 395, 677-683, 2002.

Khan, J. I., Vilmer, N., Saint-Hilaire, P., and Benz, A. O.: The solar coronal origin of slowly drifting decimetric-metric pulsation structure, A\&A, 388, 363-372, 2002.

Klassen, A., Pohjolainen, S., and Klein, K.-L.: Type II radio precursor and X-ray flare emission, Solar Phys., 218, 197-210, 2003.

Kliem, B., Karlický, M., and Benz, A. O.: Solar flare radio pulsations as a signature of dynamic magnetic reconnection, A\&A, 360, 715-728, 2000.

Kotrč, P., Karlický, M., Šimberová, S., Knížek, M, and Varady, M.: Evidence of magnetic field reconnection in the $\mathrm{H} \alpha$ eruptive prominence on 18 September 1995, Solar Phys., 182, 393-409, 1998.

Kundu, M.R., Nindos, A., Vilmer, N., Klein, K.-L., Shibata, K., and Ohyama, M.: Metric radio emission associated with X-ray plasmoid ejections, ApJ, 559, 443-451, 2001.

Kurien, S. and Sreenivasan, K. R.: Measures of anisotropy and the universal properties of turbulence, in New trends in turbulence, Les Houches Ser. LXXIV, Springer, Paris, France, 56-109, 2000.

Mészárosová, H., Veronig, A., Zlobec, P., and Karlický, M.: Analysis of solar narrow band dm-spikes observed at 1420 and 2695 MHz, A\&A, 407, 1115-1125, 2003.

Mikhailovsky, A. B.: Theory of Plasma Instabilities Vol. I, Nauka Publ., Moscow, Russia (in Russian), 1975.

Priest, E. and Forbes, T.: Magnetic Reconnection, Cambridge University Press, Cambridge, UK, 2000.

Ryabov, V. B., Stepanov, A. V., Usik, P. V., Vavriv, D. M., Vinogradov, V. V., and Yurovsky, Yu. F.: From chaotic to 1/f processes in solar mcw-bursts, A\&A, 324, 750-762, 1997.

Ohyama, M. and Shibata, K.: X-ray plasma ejection associated with an impulsive flare on 5 October 1992: Physical conditions of Xray plasma ejection, ApJ, 499, 934-944, 1998.

Sawant, H. S., Karlický, M., Fernandes, F. C. R., and Cecatto, J. R.: Observation of harmonically related solar radio zebra patterns in the 1-4 GHz frequency range, A\&A, 396, 1015-1018, 2002.

Shibata, K. and Tanuma, S.: Plasmoid-induced-reconnection and fractal reconnection, Earth Planets Space, 53, 473-482, 2001.

Tajima, T., Sakai, J.I., Nakajima, H., Kosugi, T., Brunel, F., and Kundu, M. R.: Current loop coalescence model of solar flares, ApJ, 321, 1031-1048, 1987. 
Tanuma, S., Yokoyama, T., Kudoh, T., and Shibata, K.: Twodimensional magnetohydrodynamic numerical simulations of magnetic reconnection triggered by a supernova shock in the interstellar medium: generation of X-ray gas in the galaxy, ApJ, 551, 312-332, 2001.

Valdivia, J. A., Klimas, A., Vassiliadis, D., Uritsky, V., and Takalo, J.: Self-organization in a current sheet model, Space Sci. Rev., $107,515-522,2003$
Yokoyama, T., Akita, K., Morimoto, T., Inoue, K. and Newmark, J.: Clear evidence of reconnection inflow of a solar flare, ApJL, 546, L69-L72, 2001.

Zheleznyakov, V. V. and Zlotnik, E. Y.: Cyclotron wave instability in the corona and origin of solar radio emission with fine structure, Solar Phys., 44, 461-470, 1975.

Zlobec, P. and Karlický, M.: Narrowband dm-spikes observed during the 15 June 1991 flare, Sol. Phys., 182, 477-496, 1998. 